\title{
DESIGN PARA MUDANÇA DE COMPORTAMENTO: UMA REVISÃO CRITICA
}

Juliana Gonçalves Mota

Universidade do Vale do Rio dos Sinos

julianagmota@gmail.com

Filipe Campelo Xavier da Costa

Universidade do Vale do Rio dos Sinos

fcampelo@unisinos.br

Resumo: Este artigo tem por objetivo realizar uma revisão crítica sobre as abordagens de design para mudança de comportamento e suas áreas de atuação. A partir desta análise, são discutidas oportunidades de desenvolvimento de projetos para a construção e manutenção de hábitos para o bem-estar, com foco nos processos de design orientados para a promoção do comportamento alimentar e da educação nutricional na infância. Dentre as perspectivas estudadas, o modelo proposto por Ludden e Hekkert (2014) foi assumido como o mais adequado para atender a este propósito, auxiliando na compreensão de processos de projeto para o engajamento dos usuários em práticas saudáveis.

Palavras-chave: design para mudança de comportamento, processo de projeto, comportamento nutricional infantil.

\begin{abstract}
This paper presents a critical review of design for behavior change approaches and their applications. From this perspective, project development opportunities are discussed for building and maintenance habits for well-being, especially focused on design processes oriented to the eating behavior and nutrition education in childhood. Among these perspectives, the model proposed by Ludden and Hekkert (2014) was assumed as the most suitable for this context, assisting in a better understanding of design processes for user engagement in healthy practices.
\end{abstract}

Keywords: design for behavior change, design process, nutritional child behavior 


\section{INTRODUÇÃO}

O design compreende, em sua essência, a transformação do mundo natural através da criação de artificialidades (Flusser, 2007). Sejam produtos, serviços ou ambientes, os artefatos têm implicações sobre a maneira como as pessoas pensam e interagem em seus contextos, ajudando, assim, a dar forma à cultura e às estruturas sociais (Niedderer, 2013; Tromp e Hekkert, 2014). Nesta perspectiva, o papel do design extrapola a criação de produtos, passando a interferir sobre o comportamento dos usuários e sobre a forma "como os seres humanos relacionam-se através da influência mediadora dos produtos" (Buchanan, 2001, p. 11).

Diante da manifestação de problemas sociais complexos, a capacidade de incentivar atitudes para o bem-estar tornou-se cada vez mais reconhecida como uma atribuição do design. Uma das razões para a ampliação do escopo do design deve-se ao fato de que as soluções para a grande maioria destes problemas demandam justamente mudanças de hábito dos indivíduos (Tromp et al., 2011).

Para criar artefatos com maiores chances de influenciar o comportamento, designers e pesquisadores identificaram a necessidade de entender melhor a formação dos hábitos e escolhas dos usuários. Tal condição motivou a aproximação do design a preceitos teóricos da psicologia, proporcionando a emergência de um amplo espectro de abordagens reconhecido como design para mudança de comportamento. 0 propósito deste campo consiste na compreensão mais apurada das motivações e desejos dos indivíduos e na tradução deste conhecimento em estratégias para estimular atitudes em benefício social e ambiental (Lockton et al., 2009).

Em resposta à natureza ampla e multidisciplinar do design para mudança de comportamento, Niedderer et al. (2014) elaboraram um mapeamento de suas abordagens mais relevantes. O estudo analisou também de que forma tais perspectivas associam-se às teorias comportamentais, identificando quatro áreas nas quais os projetos deste campo são potencialmente aplicados: tecnologia persuasiva, design para o comportamento sustentável, design social e contra o crime, e design para o bem-estar.

Esse artigo tem como propósito realizar uma revisão crítica sobre as abordagens de design para mudança de comportamento e discutir as oportunidades de desenvolvimento de projetos para a construção e manutenção de hábitos saudáveis. A temática corresponde a um dos eixos de investigação dentro do grupo de pesquisa ao qual pertencemos, especializado nas relações entre processos de design e a experiência do usuário. Um dos estudos em desenvolvimento visa discutir processos de design orientados para mudança de comportamento e educação nutricional infantil.

A questão da alimentação infantil e o crescimento da obesidade junto ao publico infantil têm ocupado espaço nas discussões sobre políticas de saúde publica em todo o mundo. A constatação de que os padrões nutricionais na infância têm relação direta com a saúde na vida adulta motivou a criação de diversas campanhas para incentivar a alimentação saudável entre as crianças. No entanto, os prognósticos recentes sobre o crescimento da obesidade infantil ${ }^{1}$ sugerem que tais abordagens,

\footnotetext{
${ }^{1}$ De acordo com o relatório divulgado em janeiro de 2016 pela Comissão para o Fim da Obesidade Infantil (OMS), o número de crianças obesas ou acima do peso com até cinco anos ultrapassa os 41 milhões em todo o mundo. Disponível em: <http://www.who.int/end-childhood-obesity/news/launchfinal-report/en/>. Acesso em: 28.01.2016.
} 
embora relevantes, resultam em um impacto mínimo sobre o comportamento nutricional da criança (Murillo et al., 2015). Esta constatação indica como uma alternativa para o problema o desenvolvimento de projetos que atuem principalmente sobre a motivação dos indivíduos em adotar novos hábitos para o bem-estar.

Neste contexto, o design constitui-se como um importante condutor de transformação e inovação, uma vez que possui conhecimentos que possibilitam "descobrir do que as pessoas necessitam, o que desejam, como elas interpretam os produtos que utilizam e como isso afeta as suas experiências" (Tromp, 2013). Considerando especificamente o campo do design para mudança de comportamento, seus conceitos e ferramentas auxiliam na criação de artefatos para encorajar novas atitudes nos usuários, revelando seu potencial na promoção de "futuros éticos e sustentáveis através da inovação" (Niedderer et al., 2014, p.107).

\section{DESIGN PARA MUDANCA DE COMPORTAMENTO: A EMERGENCIA DE UMA ÁREA}

Frente ao agravamento de problemas sociais nas últimas décadas, o papel estratégico do design no processo de mudança de comportamento dos indivíduos tornou-se ainda mais reconhecido (Tromp et al., 2011; Wever, 2012; Niedderer et al., 2014). Isso se justifica, uma vez que as soluções para tais problemas demandam, em sua maioria, o estímulo de atitudes entre os usuários que impactem positivamente o âmbito individual e coletivo (Tromp, 2013; Ludden e Hekkert, 2014).

Para melhor compreender como se constituem as decisões e hábitos dos usuários, designers e pesquisadores buscaram na psicologia fundamentos teóricos para desenvolver artefatos com maiores chances de influenciar o comportamento. Junto a isso, as discussões acerca das consequências éticas da ação dos designers sobre os usuários motivaram pesquisas a respeito das implicações sociais do design. Destes dois movimentos surge uma ampla série de abordagens reconhecida como design para mudança de comportamento.

A partir da revisão dos principais estudos da área, foram identificados três elementos que caracterizam as abordagens neste domínio: a base em teorias comportamentais, o emprego de métodos centrados no usuário e o reconhecimento da responsabilidade ética dos designers no processo de projeto. Cada propriedade será aprofundada a seguir.

Como princípio mais elementar, o design para mudança de comportamento busca transformar a compreensão mais profunda do ser humano e de suas formas de agir em estratégias para a criação de soluções que beneficiem os indivíduos e a sociedade (Lockton et al., 2013; Tromp e Hekkert, 2014). Para tanto, modelos e ferramentas de design foram criados com base em teorias comportamentais. À medida que tais preceitos teóricos são incorporados ao projeto, os designers têm subsídios mais qualificados para a criação de soluções que incentivem os usuários a tomar decisões para o seu bem-estar (Weinreich, 2011).

O emprego de métodos do design centrado no usuário é também uma propriedade do design para mudança de comportamento. Para Bhamra et al. (2008; 2011), é fundamental que os projetos deste campo partam de um estudo mais profundo da interação dos usuários com os artefatos e com o seu contexto social. Conforme assinala Redström (2006), devido aos usuários serem parte ativa dos sistemas, eles requerem uma compreensão mais atenta de suas experiências, motivações e necessidades. Nesse sentido, os métodos centrados no usuário são 
essenciais em projetos para a mudança de comportamento, pois constroem a base de conhecimento necessária para a criação de artefatos com mais chances de estimular novos hábitos (Wever et al., 2008).

Por fim, como terceira característica do campo, destaca-se o reconhecimento da responsabilidade dos designers sobre as práticas sociais em todo o processo de projeto (Tromp, 2013). Niedderer et al. (2014) definem este campo como uma abordagem de transformações éticas e inovadoras com impacto no presente e no futuro, que coloca no centro de suas discussões a reflexão crítica sobre o papel do designer.

Frente à multiplicidade de abordagens que compõem o design para mudança de comportamento, Niedderer et al. (2014) elaboraram um mapeamento dos principais estudos, analisando os modelos e mecanismos que sustentam cada proposta. De maneira geral, essas abordagens têm como objetivo estimular as pessoas a fazer algo ou impedi-las de tomar determinada atitude. Para este fim, as estratégias podem assumir um caráter prescritivo, criando soluções para habilitar ou dificultar o usuário a realizar uma ação. Em uma perspectiva distinta, as estratégias podem focar em mecanismos para envolvê-lo a agir (ou desistir) voluntariamente, através de incentivos ou barreiras.

A partir do mapeamento de Niedderer et al. (2014), os projetos para mudança de comportamento podem ser agrupados em quatro áreas-chave do design nas quais são predominantemente desenvolvidos: tecnologia persuasiva, design para 0 comportamento sustentável, design social e contra o crime, e design para o bem-estar. A seguir, serão detalhadas as principais abordagens de cada área e os modelos correspondentes, sintetizados na figura 1 .

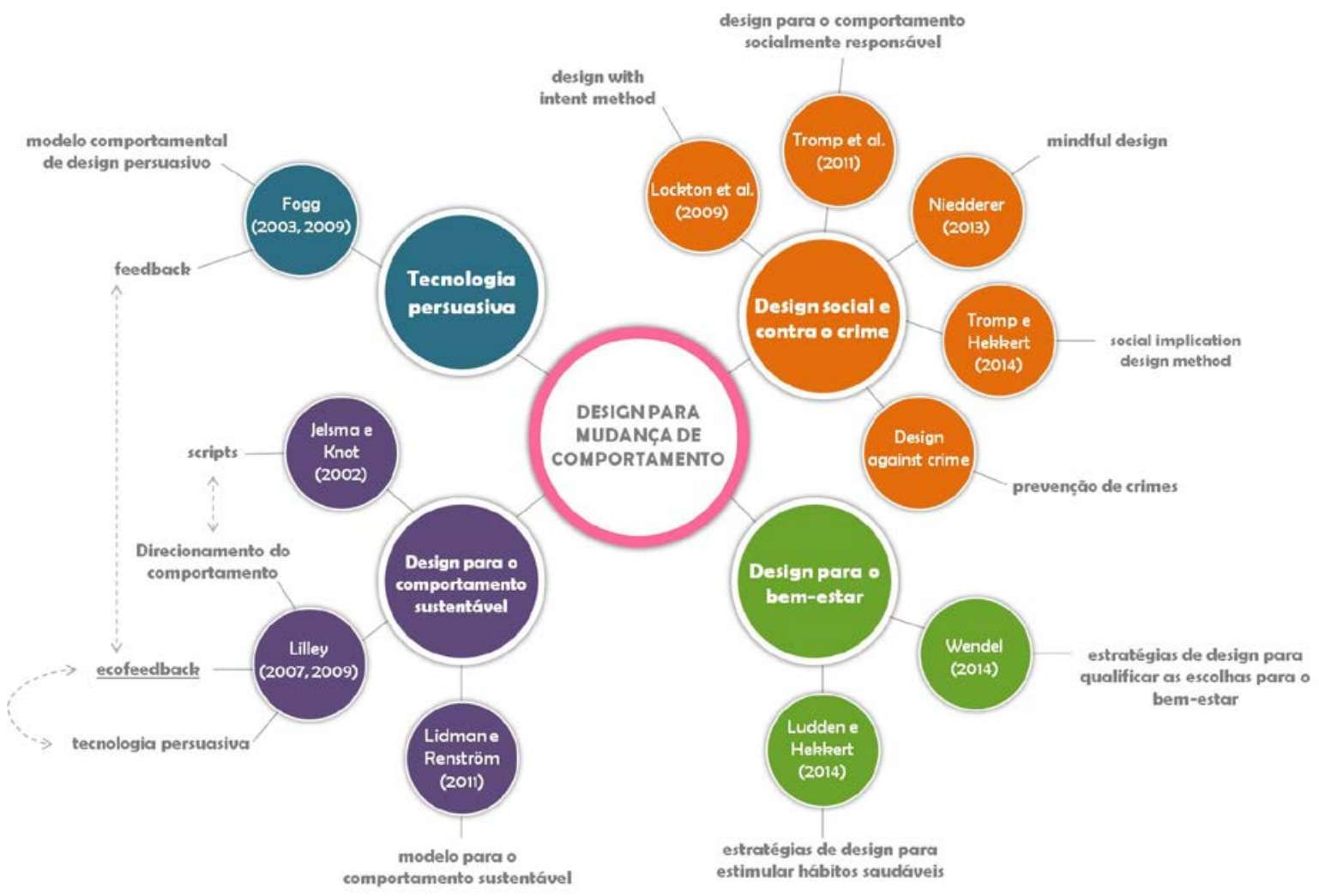

Figura 1: Principais abordagens do design para mudança de comportamento Fonte: Elaborado pelos autores 


\subsection{Tecnologia Persuasiva}

Uma das abordagens mais influentes no campo do design para mudança de comportamento diz respeito à área de tecnologia persuasiva, instituída pelos estudos de Fogg (2003). Com destaque no campo da Interação Humano-Computador, esta perspectiva considera a influência dos sistemas computacionais sobre os usuários, "tratando fundamentalmente sobre como aprender a automatizar a mudança de comportamento através da tecnologia" (Fogg, 2009). Dentre as estratégias para estimular o comportamento humano, ressalta-se o conceito de feedback, que influenciou outras abordagens, como o design para o comportamento sustentável discutido adiante.

Provindo desses estudos, o Modelo Comportamental de Design Persuasivo (Fogg, 2009) constitui-se por três variáveis interdependentes: motivação, habilidade e estímulo (Figura 2). A motivação diz respeito à disposição interna do usuário em realizar a ação, ao passo que a habilidade está relacionada ao nível de simplicidade da tarefa, revelando-se mais ou menos mais fácil de ser executada. A estrela sinaliza o comportamento-alvo que o designer espera incentivar, enquanto que o estímulo corresponde a sinais que incitam o usuário a agir no momento em que estes ocorrem.

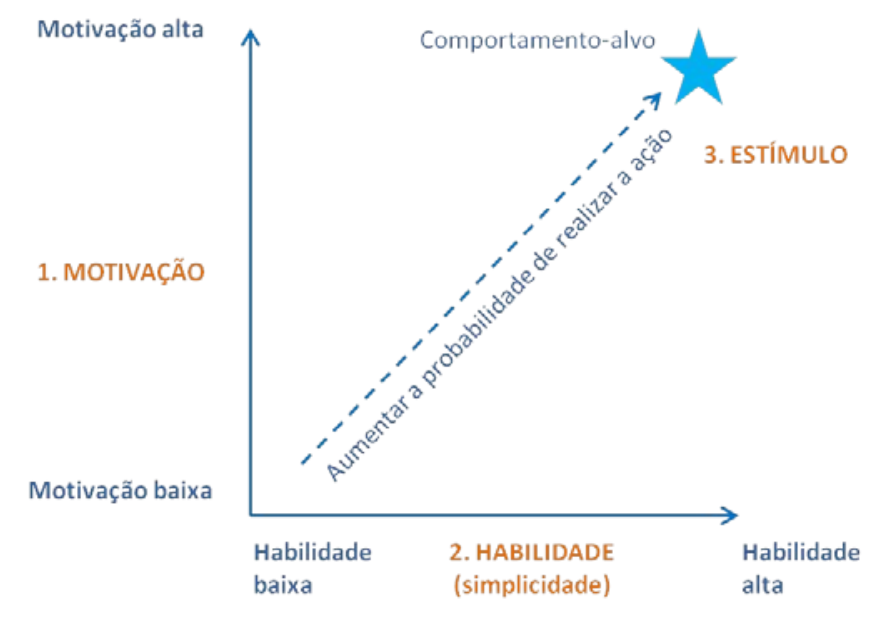

Figura 2: Modelo Comportamental de Design Persuasivo Fonte: Adaptado de Fogg (2009)

Conforme o modelo, os designers devem elevar não só a motivação do usuário, como também sua habilidade em realizar a tarefa desejada, tornando-a mais fácil de ser executada. Nesse sentido, a harmonização das três variáveis no projeto é fundamental para que o indivíduo realize o comportamento-alvo (Fogg, 2009). Tal perspectiva oferece uma compreensão sistematizada não só dos processos subjacentes à motivação dos usuários, como também das estratégias que desencadeiam novos comportamentos através de sistemas tecnológicos (Fogg, 2009).

\subsection{Design para o comportamento sustentável}

No âmbito das estratégias para estimular hábitos sustentáveis, os estudos em design para mudança de comportamento desenvolveram-se de forma significativa. Os primeiros projetos de design para sustentabilidade concentravam-se principalmente nos aspectos tecnológicos dos produtos, desconsiderando os hábitos e experiências dos usuários com os artefatos (Wever et al., 2008). Tais estratégias, entretanto, 
mostraram-se insuficientes para mudar a atitude dos indivíduos e reduzir o impacto ambiental, já que os principais efeitos dos produtos sobre o meio ambiente foram identificados na fase de uso (Bhamra et al., 2008; Wever et al., 2008). Segundo Zachrisson e Boks (2010), os estudos mais recentes na área do design para sustentabilidade apontam para a eficácia dos processos de mudança de comportamento dos usuários na redução de impactos ambientais.

Diante desta conclusão, Wever et al. (2008) sugerem que os projetos para a sustentabilidade devem associar métodos do design centrado no usuário à criação de soluções tecnológicas, para que possam qualificar as experiências de uso e incentivar atitudes sustentáveis. Esta mudança de perspectiva motivou a criação de um novo campo de estudo denominado design para o comportamento sustentável (Bhamra et al., 2011). Os pesquisadores da área entendem que os designers têm o conhecimento e as habilidades necessárias para reduzir os impactos ambientais por meio de projetos que, intencionalmente, moldem o comportamento em direção a objetivos mais sustentáveis (Wever et al., 2008; Lilley, 2009).

Jelsma e Knot (2002) concentraram sua pesquisa no conceito de script e sua aplicação em projetos de serviços sustentáveis. Segundo os pesquisadores, os scripts correspondem a regras e valores - como respeito à natureza - que são atribuídos aos artefatos através da ação intencional dos designers. Desse modo, os atributos dos produtos podem ser projetados, de forma mais ou menos explícita, para facilitar as atitudes sustentáveis dos usuários (ou para colocar entraves às ações indesejadas).

Inspirada no estudo de Jelsma e Knot e em Fogg, Lilley (2007) desenvolveu um modelo no qual identifica três intervenções que podem ser associadas à projetos para estimular hábitos em benefício ao meio ambiente (Figura 3).

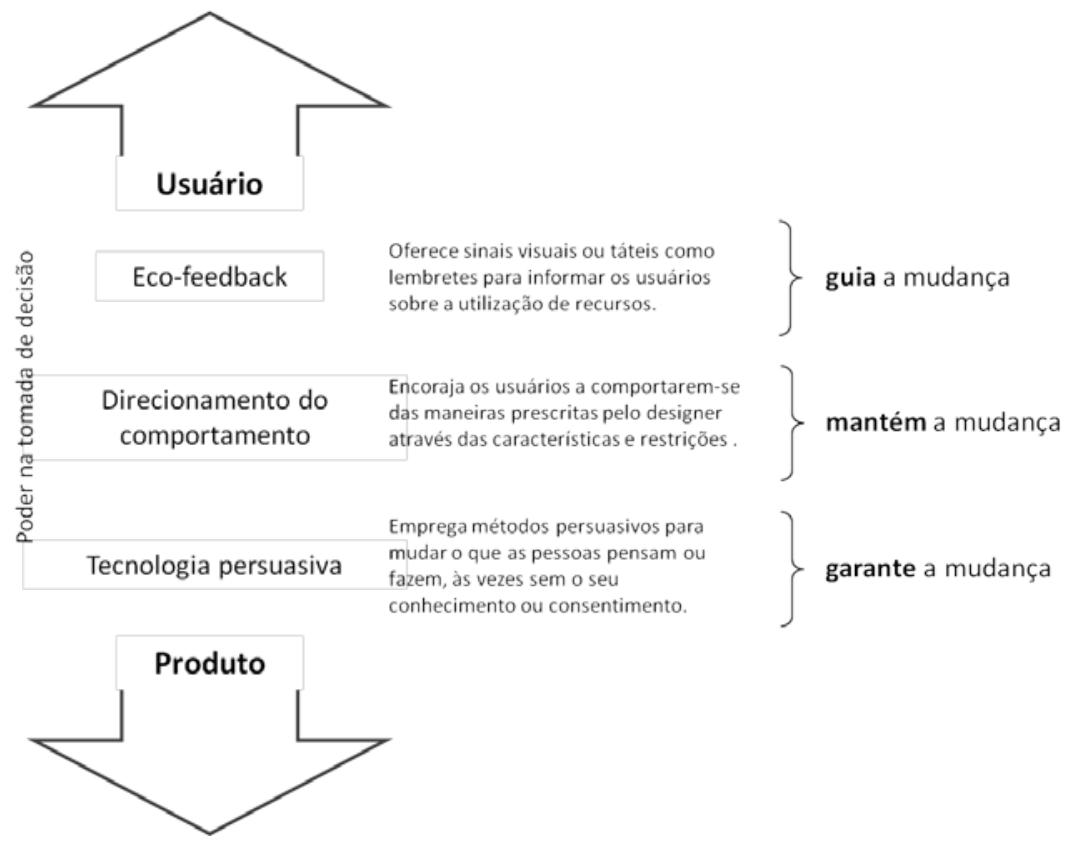

Figura 3: Modelo para o comportamento sustentável - Lilley Fonte: Lilley (2009)

A intervenção eco-feedback refere-se à possibilidade do artefato orientar o comportamento do usuário através do envio de sinais sonoros, visuais ou táteis que 
deem respostas sobre o impacto ambiental de suas ações. O direcionamento do comportamento sugere intervenções prescritas pelos designers para incentivar o indivíduo a manter seus hábitos sustentáveis. A última categoria trata de estratégias de design mais incisivas, que delegam ao artefato o papel de forçar o comportamento desejado ou de impedir as ações indesejadas (Wever et al., 2008).

Ancoradas nas propostas de Lilley (2007) e Wever et al. (2008), Lidman e Renström (2011) propuseram um método com cinco classes de estratégia de acordo com a sua função: instruir, estimular, induzir, coagir e corresponder (Figura 4). As categorias estão dispostas de forma a demonstrar a diferença de controle entre o usuário e o designer sobre o processo de mudança do comportamento. O modelo oferece uma taxonomia das estratégias, além de associar a cada uma delas técnicas para aumentar o nível de consciência do usuário acerca de seus impactos ambientais.

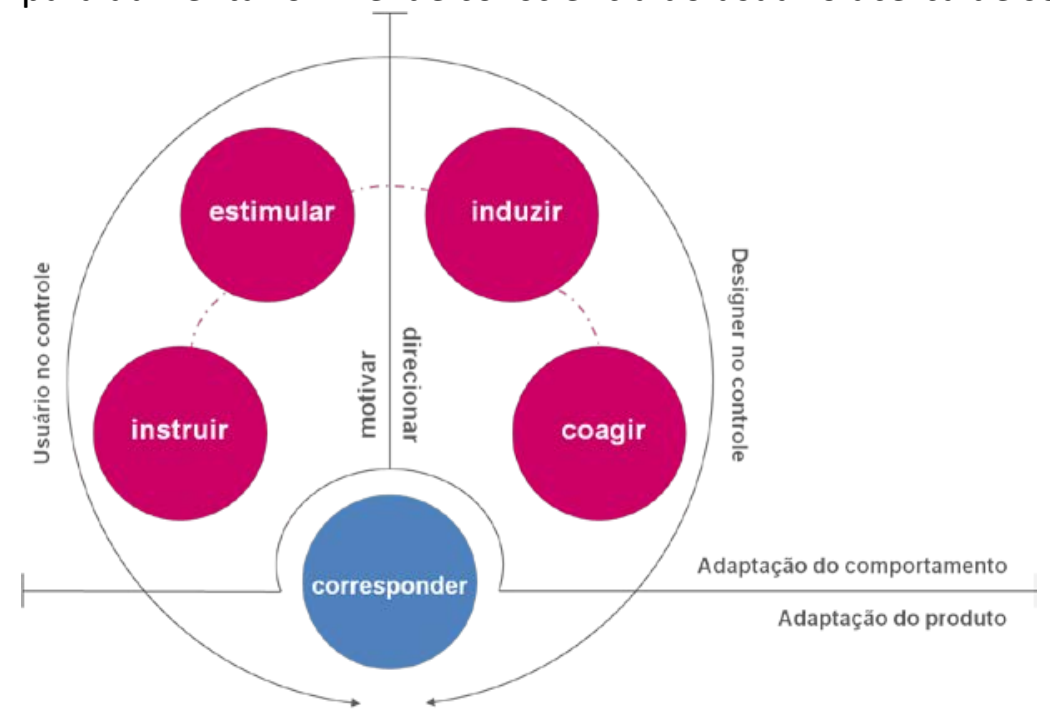

Figura 4: Modelo para o comportamento sustentável - Lidman e Renström.

Fonte: Adaptado de Lidman e Renström (2011)

\subsection{Design social e o Design contra o Crime}

Além do foco sobre as atitudes sustentáveis, o design para mudança de comportamento abrange estratégias para estimular ações socialmente responsáveis. 0 método Design with Intent oferece técnicas para reprojetar um sistema - seja um produto, serviço ou ambiente -, com o propósito de motivar, habilitar ou constranger o usuário a realizar um comportamento-alvo (Lockton et al., 2009). O método combina uma série de técnicas para incentivar novos hábitos, facilitar a realização da ação desejada ou criar entraves ao comportamento que se pretende evitar, tornando-o mais difícil de ser executado.

A abordagem de Tromp et al. (2011) propõe uma classificação da influência dos artefatos, cujo principal critério é a experiência do usuário desejada. Ao pesquisarem de que forma os produtos podem ser projetados para incentivar atitudes responsáveis, os autores categorizam as influências dos artefatos em quatro tipos: decisiva, coerciva, persuasiva e sedutora. Na base destas interferências está o seu nível de força, que pode variar entre forte ou fraco, ou ser implícito ou explícito (Tromp et al., 2011). O modelo combina cada tipo de influência com diferentes estratégias de design, sugerindo a situação de projeto mais indicada para a aplicação de cada uma. 
Desenvolvido por Niedderer (2013), o mindful design trata de estímulos para instigar a reflexão crítica dos usuários sobre suas atitudes. A abordagem tem origem na teoria da consciência plena (Langer, 1989), segundo a qual é necessário criar um estímulo para que o indivíduo reflita sobre as motivações que determinam suas ações, ajudando-o a tomar decisões mais comprometidas com seus princípios. Considerando os artefatos como agentes mediadores entre os usuários e o contexto social, Niedderer (2013) defende que o design pode modificar a percepção dos indivíduos e elevar o nível de consciência crítica sobre seus atos. Cabe aos designers, portanto, criar sistemas que deem suporte para o processo de consciência plena dos usuários e para a superação dos obstáculos para um novo comportamento.

Para auxiliar os designers na promoção de impactos sociais positivos, Tromp e Hekkert (2014) desenvolveram o método Social Implication Design (SID). Os autores entendem que as preocupações individuais muitas vezes estão em conflito com os propósitos coletivos. Neste sentido, o método propõe que o projeto se inicie pela avaliação de implicações sociais desejadas pelos designers, para então partir para a criação de um artefato que possa causar tais impactos, sugerindo um processo de design no qual os projetistas atuem como mediadores desta tensão.

Em conformidade com esta dimensão social do design, foram desenvolvidas abordagens na área de prevenção de crimes. Trata-se de projetos como o Design Against Crime ${ }^{2}$, cujo objetivo é encorajar comportamentos mais preventivos ou constranger atitudes ilícitas para promover o bem-estar social (Niedderer et al., 2014). Projetos desta natureza estão voltados à criação de produtos, serviços e plataformas, tendo como um de seus princípios o respeito às características do ambiente no qual são aplicados.

\subsection{Design para o bem-estar}

O design para mudança de comportamento orienta-se também para a promoção do bem-estar. De acordo com Wendel (2014), os artefatos podem ser desenvolvidos para dar suporte à ação dos indivíduos que não conseguem atingir objetivos pessoais, como o cuidado com a saúde ou a organização das finanças. Fundamentado em conceitos da psicologia, da tecnologia persuasiva e da economia comportamental, o método detalha as quatro fases que a atividade projetual deve conter para conduzir a mudança de comportamento.

A fase inicial do processo (compreender) tem o propósito de fundamentar o projeto com informações acerca das motivações e desejos do usuário. Com base nessa compreensão, o designer avança para a etapa seguinte (descobrir), na qual identifica o público e o comportamento-alvo do projeto. A fase de projetação envolve não só o produto, mas também o contexto no qual o indivíduo está inserido (Wendel, 2014). No quarto estágio ocorre a avaliação dos impactos do produto sobre os hábitos dos usuários, possibilitando a geração de insights para o aperfeiçoamento do projeto (Wendel, 2014, p. 201). O modelo, de natureza iterativa, sugere que os processos devem ser desenvolvidos até que a mudança de comportamento desejada seja efetivamente atingida.

Com o propósito explícito de estimular hábitos saudáveis, o modelo de Ludden e Hekkert (2014) propõe a associação de estratégias de design às etapas da

\footnotetext{
${ }^{2}$ Disponível em: <http://www.designagainstcrime.com>. Acesso em: 26.02.2016.
} 
transformação do comportamento, respeitando a disposição interna - ou o estágio motivacional - do usuário nesse processo. Os autores acreditam que, ao projetarem para cada fase de mudança comportamental, os designers ajudam a conduzir as ações dos usuários em direção a um processo de transformação mais eficaz e sustentável.

A abordagem foi criada com base no Modelo Transteórico de mudança comportamental (MTT), segundo o qual a assimilação de hábitos saudáveis e permanentes contempla cinco etapas: pré-contemplação, contemplação, preparação, ação e manutenção (Prochaska e Velicer, 1997). Cada etapa revela o nível motivacional do indivíduo no processo de mudança, compreendendo desde a tomada consciência sobre a importância da transformação até a fase em que o novo comportamento está totalmente incorporado e deve ser mantido (Prochaska e Velicer, 1997).

No estágio de pré-contemplação, os indivíduos ainda não estão cientes das consequências de suas atitudes atuais e da necessidade da mudança. Na fase seguinte, manifestam-se as primeiras intenções de transformação, e os benefícios de um novo comportamento tornam-se mais evidentes. A intenção de mudar emerge no estágio de preparação, quando as pessoas já têm em mente um plano de ação. A ação, por sua vez, compreende o período em que o novo comportamento será realizado no curto prazo. E, por fim, na fase de manutenção, o processo de mudança já está instituído, sustentando-se por um período acima de seis meses (Prochaska e Velicer, 1997).

A construção das bases para o engajamento do usuário na mudança de comportamento ocorre nas primeiras três etapas, ao passo que, nas duas últimas, o indivíduo assume o controle da ação (Ludden e Hekkert, 2014). Além das cinco etapas de transformação dos hábitos, Prochaska et al. (1992) apontam dez processos que ajudam a compreender como ocorrem as mudanças de comportamento. Trata-se de processos como o aumento de consciência ou a auto-reavaliação do indivíduo, que, no modelo analisado, têm a função de dar suporte a projetos para auxiliar o usuário no engajamento em uma atitude para o bem-estar. Seguindo esta teoria, o modelo de Ludden e Hekkert combina os processos e fases do MTT a quatro etapas de projeto, de acordo com o seu objetivo: sensibilização, capacitação, motivação e atenuação gradual da intervenção (Figura 5).

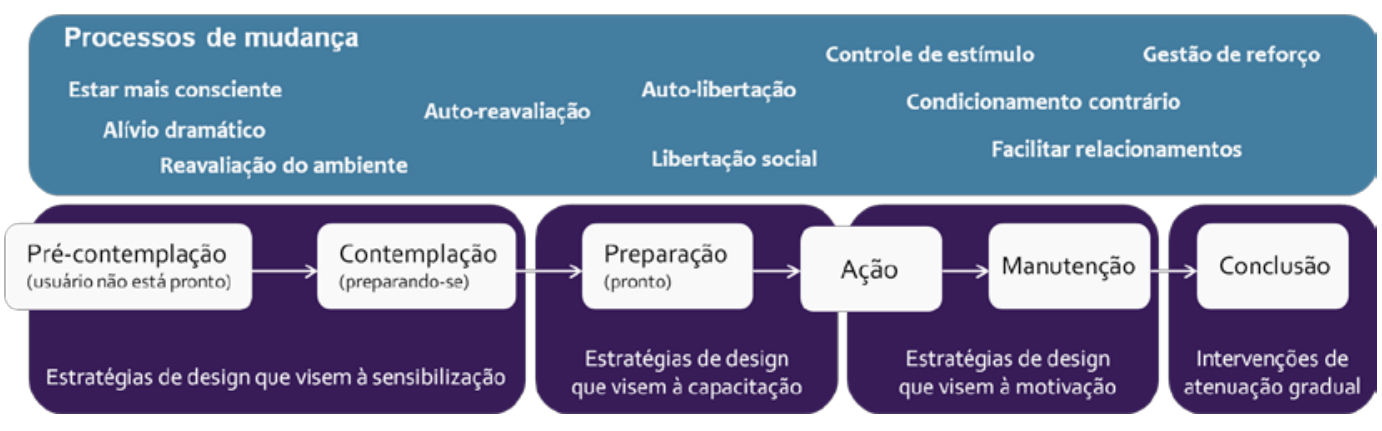

Figura 5: Modelo para o comportamento saudável.

Fonte: Adaptado de Ludden e Hekkert (2014)

As estratégias para sensibilização buscam ajudar o indivíduo a avaliar suas atuais escolhas e os benefícios dos novos hábitos, aumentando seu nível de consciência acerca da mudança. Em projetos para o estímulo de hábitos saudáveis, Ludden e Hekkert (2014) sugerem que tais intervenções devem apresentar as duas 
possibilidades de escolha ao usuário (a opção saudável e a prejudicial à saúde), tornando a escolha saudável mais fácil de ser realizada. Nesta categoria, os autores citam uma máquina automática de lanches projetada para incentivar o consumo de alimentos saudáveis no ambiente de trabalho. A máquina foi desenvolvida de maneira que a opção pelas frutas se tornasse mais acessível ao usuário.

As intervenções de capacitação dão suporte para que as pessoas criem seu próprio plano de ação (Niedderer et al., 2014). Como exemplo desta categoria, apresenta-se o projeto de um restaurante que, por meio do código das cores de seus utensílios, comunica ao usuário o valor nutricional do alimento correspondente e a quantidade que eles devem ser consumidos. As colheres verdes informam que os respectivos pratos podem ser ingeridos sem restrição. Alimentos com a colher amarela indicam consumo moderado, ao passo que as comidas servidas com um utensílio vermelho devem ser ingeridas em menor quantidade (Ludden e Hekkert, 2014).

No que diz respeito à motivação, os designers devem criar mecanismos que auxiliem na manutenção dos novos comportamentos, ajudando o usuário a evitar os antigos hábitos. Para este fim, a Philips desenvolveu um monitor pessoal de atividades físicas que ajuda a manter o controle do comportamento. O sistema oferece sugestões de atividades personalizadas, a partir dos dados coletados durante a rotina do usuário (Ludden e Hekkert, 2014).

Com o objetivo já alcançado, os designers devem projetar intervenções que reforcem o novo hábito, mas de forma a atenuarem-se gradualmente para que o usuário assuma a autonomia no processo. Um exemplo de artefato desenvolvido a partir destas estratégias é o Mother Sense ${ }^{3}$, um sistema inteligente que analisa os movimentos e atividades de rotina do usuário, oferecendo a ele lembretes por meio de um aplicativo para smartphone para que mantenha o comportamento desejado.

Através do modelo, é possível identificar o estado motivacional do usuário em relação à mudança de comportamento, associando a cada etapa estratégias de design para impulsionar o indivíduo. Tal associação é fundamental, uma vez que as intervenções de design para mudança de comportamento somente serão aceitas pelos usuários se elas estiverem combinadas com o seu estado de motivação neste processo (Ludden e Hekkert, 2014).

\section{CONSIDERAÇÕES FINAIS}

Esse artigo tem como propósito realizar uma revisão crítica sobre as abordagens de design para mudança de comportamento e seus campos de atuação. A evolução do desenvolvimento de estratégias projetuais focadas na proposição de artefatos capazes de influenciar a mudança de comportamento ou, pelo menos, de instigar o questionamento de hábitos dos indivíduos, vem se constituindo em um espaço de investigação crescente na área do design. O próprio relatório de pesquisa desenvolvido por Niedderer et al. (2014) reuniu profissionais de oito universidades europeias para identificar práticas e modelos empregados por stakeholders públicos e privados. A temática vem ganhando relevância nos congressos da área (IASDR, Design Conference, Design \& Emotion, etc.), com números crescentes de estudos sobre as distintas abordagens. Na 20 a Conferência da Design Research Society, realizado em junho de 2016 em Brighton (Reino Unido), um Special Interest Group (SIG) foi gerado

\footnotetext{
${ }^{3}$ Disponível em: <http://sen.se/mother/>. Acesso em: 21.03.2016.
} 
para acolher os esforços e trazer maior indicativos de uma agenda de pesquisa sobre a temática.

A discussão dessas abordagens, bem como sucessivas aplicações e aprimoramentos desses processos projetuais orientados para mudanças de comportamento, consiste no principal desafio a ser enfrentado. Como mencionado anteriormente, os autores desse trabalho desenvolvem uma investigação para compreender o desenvolvimento de estratégias projetuais para promover o comportamento nutricional na infância. Após a análise das possibilidades de modelos que poderiam ser empregados, foi assumido que o modelo proposto por Ludden e Hekkert, focados no design para o bem-estar, como o mais adequado para respondêlo. Esta escolha se justifica, na medida em que as intervenções de design sugeridas pelos autores ajudam a balizar a atividade projetual para o bem-estar, colocando as motivações do usuário no centro do projeto. Ao associar ferramentas do design a conceitos da psicologia para a saúde, este modelo pode contribuir com uma compreensão mais apurada a respeito dos usuários e de como se deve projetar para engajá-los em práticas saudáveis.

\section{REFERÊNCIAS}

BHAMRA, T. A.; LILLEY, D.; TANG, T. (2008) Sustainable use: changing consumer behaviour through product design. In: C. Cipolla e P.P. Peruccio (eds). Changing the Change: Design, Visions, Proposals and Tools: Proceedings. Torino, Italy.

BHAMRA, T.; LILLEY, D.; TANG, T. (2011) Design for sustainable behaviour: using products to change consumer behaviour. The Design Journal, 14, pp. 427-445.

BUCHANAN, R. (2001) Design Research and the New Learning. Design Issues, v.17, n.4, May 04, pp. 3-23.

FLUSSER, V. O mundo codificado. Por uma Filosofia do design e da comunicação (1ạ ed.) São Paulo: Cosac Naify, 2007.

FOGG, B J. (2009) A behavior model for persuasive design. In: Proceedings of the 4th international Conference on Persuasive Technology. ACM, artigo 40.

FOGG, B J. Persuasive technology: using computers to change what we think and do. Morgan Kaufmann, San Francisco, 2003.

JELSMA, J.; KNOT, M. (2002). Designing environmentally efficient services; a 'script' approach. The Journal of Sustainable Product Design 2, pp. 119-130.

LANGER, E. Mindfulness. Reading, MA: Addison Wesley, 1989.

LIDMAN, K.; RENSTRÖM, S. (2011). How to design for sustainable behaviour? A review of design strategies and an empirical study of four product concepts. (Master thesis). Göteborg, Sweden: Chalmers University of Technology.

LILLEY, D. (2007) Designing for behavioural change: reducing the social impacts of product use through design, doctoral thesis, Department of Design and Technology, Loughborough University, UK.

LILLEY, D. (2009) Design for sustainable behaviour: strategies and perceptions, Design Studies, v. 30, n. 6, pp.704-720. 
LOCKTON, D.; HARRISON, D.; STANTON, N. (2009) The design with intent method: a design tool for influencing user behavior. Applied Ergonomics, v. 41, n. 3, pp. 382392.

LOCKTON, D.; HARRISON, D.; STANTON, N. (2013) Exploring Design Patterns for Sustainable Behaviour. The Design Journal, v. 16, issue 4, pp. 431-459.

LUDDEN, G.; HEKKERT, P. (2014) Design for healthy behavior design interventions and stages of change. In: Salamanca, J., Desmet, P., Burbano, A., Ludden, G., Maya, J. (Eds.). Proceedings of the Colors of Care: The 9th International Conference on Design \& Emotion. Ediciones Uniandes, Bogotá, pp. 482-488.

MURILLO, A.; SAFAN, M.; CASTILLO-CHAVEZ, C.; CAPALDI-PHILLIPS, E.; WADHERA, D. (2015) Modeling Eating Behaviors: the Role of Environment and Positive Food Association Learning via a Ratatouille Effect. arXiv:1601.03419.

NIEDDERER, K. (2013) Mindful Design as a Driver for Social Behaviour Change. In: Proceedings of the IASDR Conference. Tokyo, Japan: IASDR.

NIEDDERER, K., MACKRILL, J., CLUNE, S., LOCKTON, D., LUDDEN, G., MORRIS, A., CAIN, R., GARDINER, E., GUTTERIDGE, R., EVANS, M., HEKKERT, P. (2014). Creating Sustainable Innovation through Design for Behaviour Change: Full Report. University of Wolverhampton, Project Partners \& AHRC.

PROCHASKA, J. O.; DICLEMENTE, C. C.; NORCROSS, J. C. (1992) In search of how people change. American Psychologist, 47, pp. 1102-1114.

PROCHASKA, J. O.; VELICER, W. F. (1997) The Transtheoretical Model of Health Behavior Change. American Journal of Health Promotion, 12, (1), pp.38-48.

REDSTRÖM, J. (2006) Towards user design? On the shift from object to user as the subject of design. Design Studies, v. 27, n. 2, march, pp. 123-139.

TROMP, N. (2013). Social Design: How products and services can help us act in ways that benefit society, Ph.D.thesis, Delft University of Technology.

TROMP, N.; HEKKERT, P. (2014) Social Implication Design (SID) - A design method to exploit the unique value of the artifact to counteract social problems. In: Proceedings of DRS2014, Umea, Sweden.

TROMP, N.; HEKKERT, P.; VERBEEK, P. (2011) Design for Socially Responsible Behavior: A Classification of Influence Based on Intended User Experience. MIT, Design Issues: v. 27, n. 3, summer, pp. 3-19.

WEINREICH, N. K. Hands-on social marketing: a step-by-step guide to designing change for good. Thousand Oaks, Calif.: SAGE Publications, 2011.

WENDEL, S. Designing for Behavior Change. O’Reilly Media, Inc., 2014.

WEVER, R. (2012) Editorial: Special Issue on Design Research for Sustainable Behaviour. Journal of Design Research, v. 10, n. 1/2.

WEVER, R.; VAN KUIJK, J.; BOKS, C. (2008) User-centered design for sustainable behaviour. International Journal of Sustainable Engineering, 1 (1), pp. 9-20. 
ZACHRISSON, J.; BOKS, C. (2010) When to apply different design for sustainable behavior strategies?. In: Proceedings of Knowledge Collaboration \& Learning for Sustainable Innovation ERSCP-EMSU Conference, Delft, The Netherlands, pp. 25-29. 